\title{
The limited influence of transference number on the performance of nanoscale batteries
}

\author{
Kaitlyn A. Dwelle and Adam P. Willard* \\ Department of Chemistry, Massachusetts Institute of Technology, Cambridge, MA 02139 \\ E-mail: awillard@mit.edu
}

\begin{abstract}
This manuscript presents a simulation study aimed at evaluating the role of transference number in determining the performance of a nanoscale Li-ion-type battery system. We employ a simulation model that supports the electrochemically driven flow of cations through a model polymer electrolyte and into a composite electrodeelectrolyte cathode. By independently varying the mobility of cations and anions, we evaluate the effect of transference number on model device performance. We find that unlike macroscopic device models, the performance of nanoscale batteries is insensitive to the value of transference number. We attribute this insensitivity to the ability of the electrolyte to support deviations from charge neutrality over nanometer length scales. We conclude that the key to performance in nanoscale battery architectures is to simply maximize cation mobility.
\end{abstract}

\section{Keywords}

transference number 


\section{Introduction}

The performance of a lithium-ion battery depends significantly on the properties of the electrolyte that mediates the flow of ions from one electrode to another. Along with chemical stability and conductivity, the transference number, $t_{+}$, has been considered an important property to optimize in order to develop high performing battery systems. ${ }^{1,2}$ In electrolytes for Li-ion batteries, $t_{+}$defines the fraction of overall ionic conductivity that comes from $\mathrm{Li}^{+} .3$ Electrolytes with high transference numbers (i.e., $t_{+} \approx 1$ ) are understood to withstand the negative effects of concentration polarization, whereby the intercolation/deintercolation of $\mathrm{Li}^{+}$at the electrodes lead to spatial gradients in the net ionic concentration of the electrolyte. ${ }^{4-6}$ In this work, we use atomistic simulations to investigate the influence of $t_{+}$on the performance of batteries with nanoscale architectures. We find that over length scales of $\sim 10 \mathrm{~nm}$, the tendency for concentration polarization gives rise to local deviations from charge neutrality that serve to mitigate the predicted performance loss. By analyzing the results of many simulations, we demonstrate that cation mobility, not the transference number, is the major predictor for model device performance and that even in cases where concentration polarization is reduced, very immobile anions can slow cation dynamics and lead to poor overall performance even at high transference numbers.

Transference number is difficult to measure experimentally, because it requires a deconvolution of a collective property (conductivity) into its microscopic components. Most popular methods, such as the Bruce \& Vincent method, ${ }^{7}$ or diffusion NMR techniques, ${ }^{8}$ express the transference number as a ratio of cation and anion diffusivities,

$$
t_{+}=\frac{D_{+}}{D_{-}+D_{+}}
$$

where $D_{+}$and $D_{-}$are the diffusion coefficients for cations and anions, respectively. Notably, this expression is based on the assumption of an ideal dilute solution of only two charge carrying species. More sophisticated corrections ${ }^{9,10}$ require the measurement of ad- 
ditional properties like the salt activity coefficient or the diffusion coefficients. In reality, most reported transference numbers are inaccurate due to non-idealities introduced by the concentrated solutions present in real electrolytes. ${ }^{2,11}$

Due to the difficulty of accurately measuring and systematically controlling $t_{+}$, most insight about its impact on device performance has been derived from theoretical or simulationbased studies. Newman and coworkers published a one-dimensional continuum model of a battery system like the one shown in Fig. 1, and showed that over macroscopic length-scales, concentration polarization can be limited or even eliminated for very high transference number systems $\left(t_{+}>.99\right) .{ }^{1}$ This is due to the fact that at very high transference numbers, the anion density is essentially immobile on the timescale of cation motion and battery discharge, and over macroscopic length scales, charge neutrality is maintained and thus the cation concentration is driven strongly to match the (flat) anion profile, as shown in Fig. 2b. As interest in nanometer scale batteries increases, it it important to understand how macroscopic theories translate to nanoscale systems. ${ }^{12}$ Over nanometer length scales, charge neutrality can be broken, especially near the interface where charge buildup can be stabilized by image charge interactions. While this electrified interface is very small on the scale of macroscpoic systems, it may span a large fraction of the electrolyte in ultra-thin systems where unscreened electric fields have already been shown to lead to enhanced transport under certain conditions. ${ }^{13}$ While the magnitude and direction of this field depends on the exact concentration and strength of the active and supporting electrolyte, this field enhancement of transport suggests that the transport parameters that lead to increased performance ultra-thin electrolytes may be fundamentally different than in macroscopic systems. Our simulations find that for ultrathin cells $(14 \mathrm{~nm})$, charge polarization is relatively unaffected by transference number, having no observable change in Coulomb-enhanced transport with an increasing transference number. In fact, there is evidence that battery performance can be hindered at very high transference number due to the current-carrying cations' attractive interactions with relatively immobile anions. 


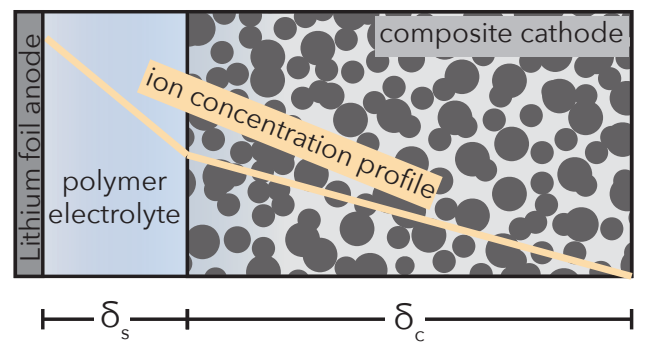

Figure 1: Diagram of the system under consideration: a battery cell with a lithium foil anode, polymer electrolyte separator, and composite cathode composed of cathode particles surrounded by electrolyte matrix. Electrochemical reactions can take place at the anode interface and throughout the composite cathode region.

We explore the role of transference number in the performance of simulated nanoscale battery systems. The details of our simulation methods are described in the following section. Then, in the Results and Discussion section, we discuss the results our simulations and their physical implications.

\section{Methods}

\section{Dynamics}

The battery model is an extension of the electrochemically active molecular dynamics methodology developed by Dwelle et. al. ${ }^{14}$ The cations and anions are explicitly simulated as Lenard-Jones particles with $\sigma=1.4 \AA$ and $\epsilon=0.4 \mathrm{kcal} / \mathrm{mol}$, and mass of $7 \mathrm{~g} / \mathrm{mol}$. Cations and anions were given point charges of +1 and -1 elementary charge respectively. All particle mobilities are tuned separately by changing the damping of the Langevin thermostat. As was done in the contiunuum model by Newman and coworkers, the dynamics in the porous cathode $\left(\delta_{s}<x<\delta_{c}\right)$ are simulated via an effective diffusivity,

$$
D_{\text {eff }}=D \epsilon^{0.5}
$$




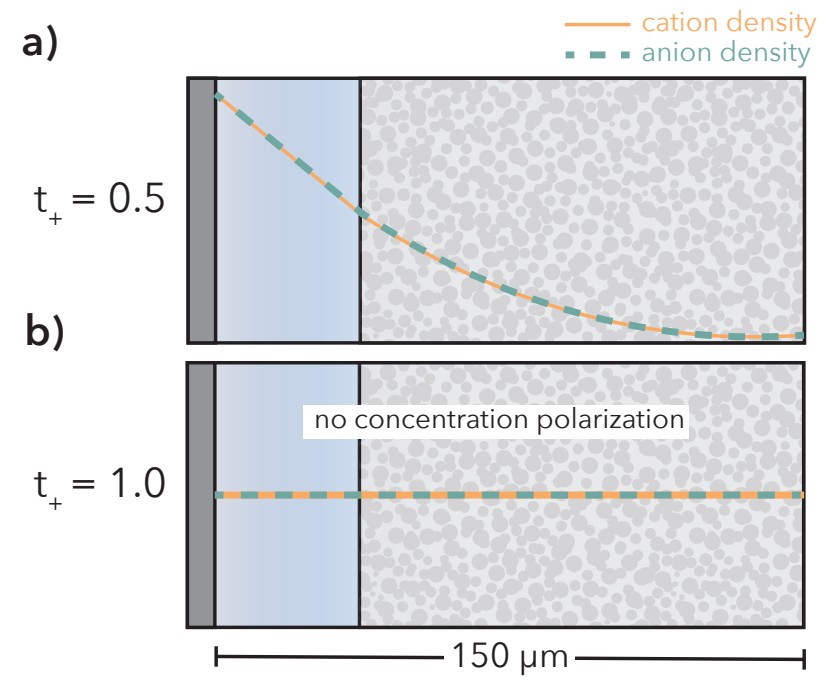

c)

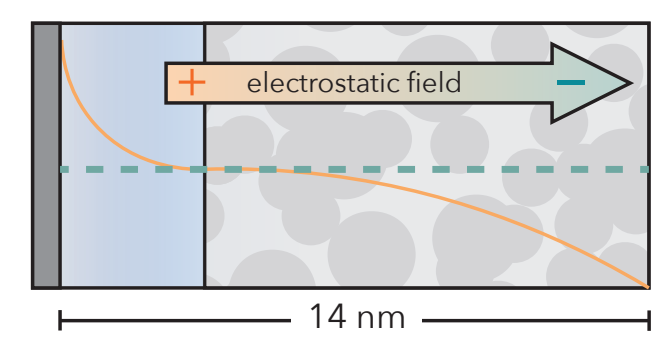

Figure 2: Comparison of concentration profiles for nanometer and micrometer scale battery systems. a) At moderate $t_{+}$, large cells experience concentration polarization, both within the electrolyte and the cathode. b) At $t_{+}=1$ and over micrometer lengthscales, the cell is locally electroneutral. Thus, the fixed, flat concentration profile of the counter-ions prevents cation polarization. This regime occurs for macroscopic cells where the bulk behavior dominates in comparison to the double layer regime. c) Over nanometer lengthscales, electroneutrality can be broken, especially near the interfaces where charged layers can be supported by image charge interactions. In this case anion polarization is determined by the free energy trade-off between an entropically favored flat profile, and enthalpically favored screening of cation polarization. At low enough concentration and small enough lengthscales, entropic considerations dominate regardless of the value of $t_{+}$. 
where $\mathrm{D}$ is the diffusivity in the electrolyte region, and $\epsilon$ is the porosity of the cathode, taken to be 0.3 in this work. Thus, the strength of the damping on each particle is a function of its location in the simulation box. The simulation box was $7 \mathrm{~nm}$ by $7 \mathrm{~nm}$ by $140 \mathrm{~nm}$ with the porus cathode region beginning at $42 \mathrm{~nm}$.

\section{Simulating Reactivity}

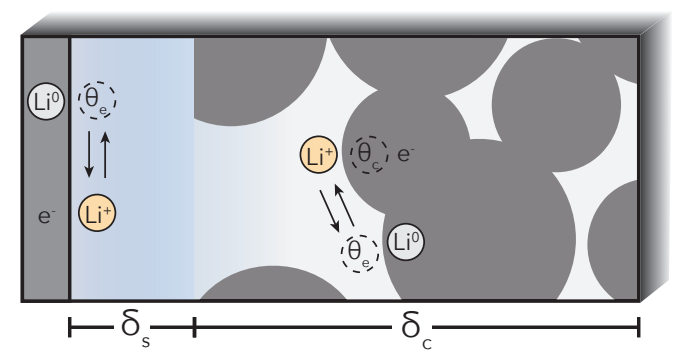

Figure 3: Caption

The active electrochemistry is described by two equilibria, one at the anode and the other in the porus cathode, as shown in figure 3. At the foil anode, the equilibrium is given by

$$
\mathrm{Li}(\text { intercalated })+\Theta_{e} \rightleftharpoons \mathrm{Li}^{+}-\Theta_{e}+\mathrm{e}^{-}
$$

where $\Theta_{e}$ is a site in the electrolyte (e.g. a coordinating site in an ion conducting polymer) and $\mathrm{Li}^{+}-\Theta_{e}$ is a site occupied by a lithium ion. This corresponds to an equilibrium between unoccupied and occupied electrolyte sites. The dynamics of the $\mathrm{Li}^{+}$in solution are simulated explicitly, while the electron and the intercalated $\mathrm{Li}^{0}$ are only included through the effects of the applied voltage on the equilibrium. In the composite cathode, the intercalation process is described by

$$
\mathrm{Li}^{+}-\Theta_{e}+\Theta_{c}+\mathrm{e}^{-} \rightleftharpoons \mathrm{Li}-\Theta_{c}+\Theta_{e}
$$

where $\Theta_{c}$ is an unoccupied cathode intercalation site, and $\mathrm{Li}-\Theta_{c}$ is an occupied site. This describes an equilibrium between the occupied electrolyte site and the occupied cathode site. The number of cathode sites is based on the stoichiometry 


\section{Defining transference number}

The transference number, $t_{+}$, is defined as the fraction of current carried by a given species in a system with no concentration gradients. ${ }^{3,15}$ Since Ohm's law relates current and conductivity, ( $i=\sigma E$, where $i$ is current, $\sigma$ is conductivity, and $E$ is the electric field), we can write $t_{+}$as

$$
t_{+}=\frac{\sigma_{+}}{\sum \sigma_{i}}
$$

where the sum is over all species in the system. For simplicity, we will only consider binary salt systems here with no supporting electrolyte so $\sum \sigma_{i}=\sigma_{+}+\sigma_{-}=\sigma$. In our simulations we will be tuning the electrophoretic mobility, $\mu_{i}$, which determines how susceptible a species is to motion under an electric field $\left(v_{i}=\mu_{i} E\right.$ where $v_{i}$ is the velocity). The relationship between conductivity, $\sigma$, and mobility, $\mu$ is given by

$$
\sigma=F \sum_{i} z_{i} \mu_{i} c_{i}
$$

where $F$ is Faraday's constant, $z_{i}$ is the charge on species $i$ and $c_{i}$ is the concentration of species $i$. The transference number can then be written as

$$
t_{+}=\frac{\sigma_{+}}{\sigma}=\frac{z_{+} \mu_{+} c_{+}}{\sum_{i} z_{i} \mu_{i} c_{i}}=\frac{\mu_{+}}{\mu_{+}+\mu_{-}}
$$

where the last equality is for a monovalent, binary electrolyte under the assumption of electroneutrality $\left(\sum z_{i} c_{i}=0\right)$. Thus by tuning the mobility of the anions and cations separately, we can directly control the expected transference number of the simulations and measure the power output as a function of transference number. 


\section{Results and Discussion}

We use electrochemically-active molecular dynamics ${ }^{14}$ to simulate a variety of cation and anion mobilities in an active electrochemical cell. Building off the work of Newman and coworkers, ${ }^{1,16}$ we simulate a battery cell with a lithium foil anode, polymer electrolyte separator, and composite cathode composed of cathode particles surrounded by electrolyte matrix. Figure 1 shows a cartoon of this system as well as an example of ion concentration which is polarized across the cell. Simulations are run using a constant voltage procedure where there is initially no lithium in the cathode, and a constant voltage is applied across the cell to drive the oxidation of lithium from the foil anode, across the electrolyte separator, and into the composite cathode where it can be reduced. Although the total power capacity of a real battery is determined by the amount of lithium which can be intercalated into the cathode before the voltage at constant current drops below some threshold, the work of Newman and coworkers showed that the increased capacity of high transference number batteries is due to changes in concentration polarization which can be evaluated during steady-state discharge. We consider the power output of our model battery under pseudo-steady-state conditions, where current is constant under an applied voltage, and favorable changes in the concentration profile manifest as higher current under the same applied voltage.

By running simulations over a range of cation and anion mobilities, we were able to capture behavior spanning from $t_{+}=0.008$ to $t_{+}=0.9992$ and a 14 -fold difference in output current density. Figure 4 shows the steady-state current density as a function of transference number. Dashed lines connect simulations at a constant $\mu_{-}$which suggest that the systematic improvement in power density with increased transference number is the result of higher cation mobility, an expected correlation since it is obvious that better battery performance is correlated with a more mobile active species. Solid lines connect simulations at a constant $\mu_{+}$, i.e. where higher transference number is the result of slowing the anionic species. In these simulations, we see no evidence of power enhancement, even at extreme transference numbers. In fact, nearly immobile anions seem to be correlated with 
slower cation dynamics.

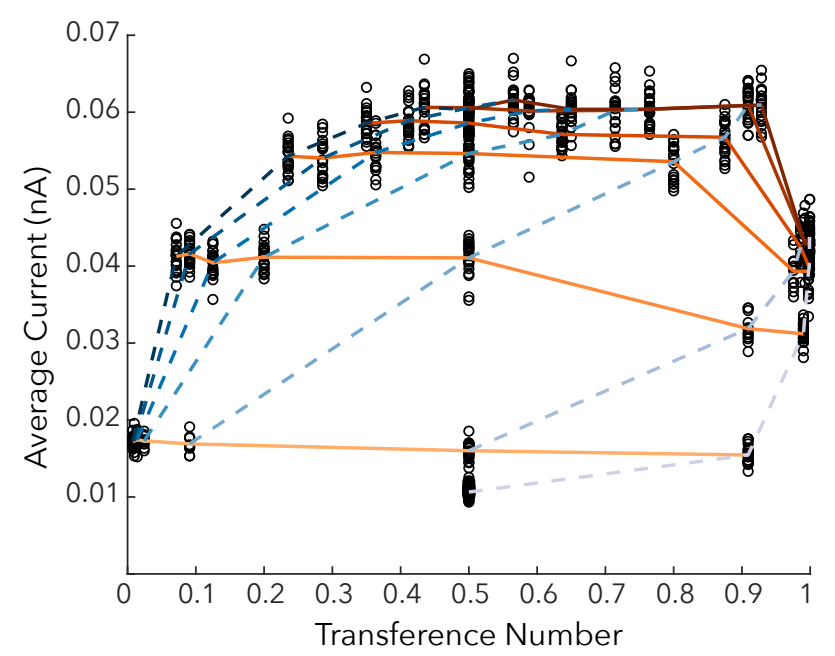

Figure 4: Average current as a function of transference number. Black circles are the result of a simulation and lines connect the means of each parameter set. Solid orange lines connect points at a constant $\mu_{+}$with darker lines having a higher value of $\mu_{+}$. Blue dashed lines connect simulations at a constant $\mu_{-}$with darker lines having a higher value of $\mu_{-}$. Values of $\mu_{+}$are sampled at values of 0.17 (lightest orange), 1.7, 6.6, 11.5, 16.5, and 21.5 (darkest orange) $\mu^{2} \mathrm{~V}^{-1} \mathrm{~s}^{-1}$ and values of $\mu_{-}$are sampled at values of 0.02 (lightest blue), $0.17,1.7$, $6.6,11.5,16.5$, and 21.5 (darkest blue) $\mu^{2} \mathrm{~V}^{-1} \mathrm{~s}^{-1}$.

Because transference number is inherently a convolution of two transport properties, the cation and anion mobilities, we have also shown the same data as a function of $\mu_{-}$and $\mu_{+}$in Fig. 5. The data plotted with solid orange lines show that extremely slow anion populations (below values of $5 \mu \mathrm{m} \mathrm{V}^{-1} \mathrm{~s}^{-1}$ ) actually decrease the battery performance, presumably due to cation-anion attractive interactions. Because of the attractive Coulomb interactions, cations may experience a type of Coulombic friction at very slow anion mobilities. This is actually consistent with many experimental measurements where limiting the counterion mobility leads to a decrease in overall conductivity. ${ }^{17}$

The right panel of figure 5 shows a monotonic increase in battery performance as cation mobility is increased as expected. The trend levels off at higher cation mobilities because the system transitions from a diffusion-limited current where an increase in cation mobility leads to a large change in the output current to a charge-transfer-limited current regime which is relatively insensitive to changes in the cation mobility. 


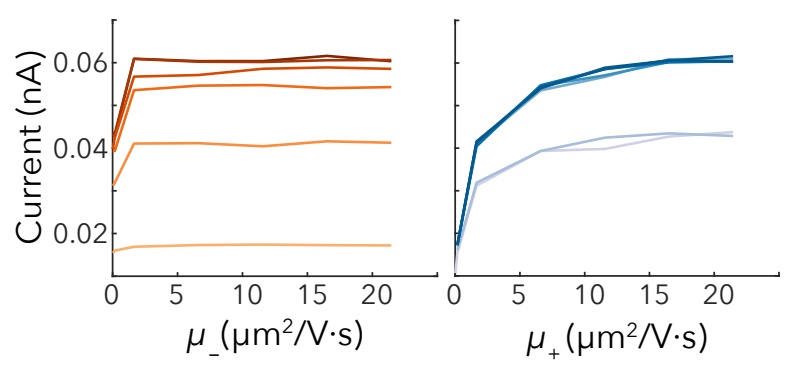

Figure 5: Average power density as a function of ion mobilities. Left: orange lines show the power density as a function of anion mobility. Darker lines correspond to a higher cation mobility. Specific line colors correspond to those in Fig. 4. Right: blue lines show the power density as a function of cation mobility. Darker lines correspond to a higher cation mobility.
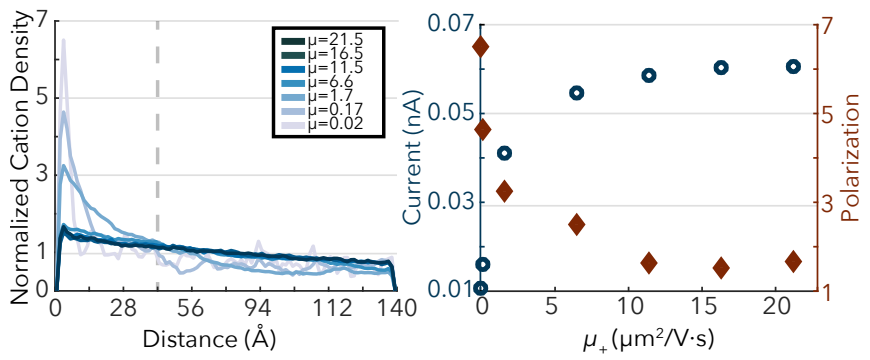

Figure 6: Left: Cation concentration profiles as a function of distance from the left foil anode over a range of cation mobilities at fixed $t_{+}=0.5$. The grey dashed line indicates the boundary between the electrolyte and composite cathode regions. Concentrations are plotted for $\mu_{+}=\mu_{-}$valued at 0.02 (lightest blue), 0.17, 1.7, 6.6, 11.5, 16.5, and 21.5 (darkest blue) $\mu^{2} \mathrm{~V}^{-1} \mathrm{~s}^{-1}$. Right: Power density (blue circles) and polarization (orange diamonds) as a function of cation mobility for the same systems plotted to the left. Polarization is plotted as the maximum normalized cation density observed near the foil anode. 
Plotting the concentration profiles at a fixed transference number of $t_{+}=0.5$, but varying cation mobility shows that systems with a higher cation mobility have lower concentration polarization and lead to better battery performance. Figure 6 contains plots of the concentration profiles and the power densities and maximum cation densities (a proxy for concentration polarization). The right-hand panel of Fig. 6 shows that lower cation polarization correlates well with higher power density. This gives a good example of a situation where optimizing the cation mobility, not the transference number, leads to increased performance.

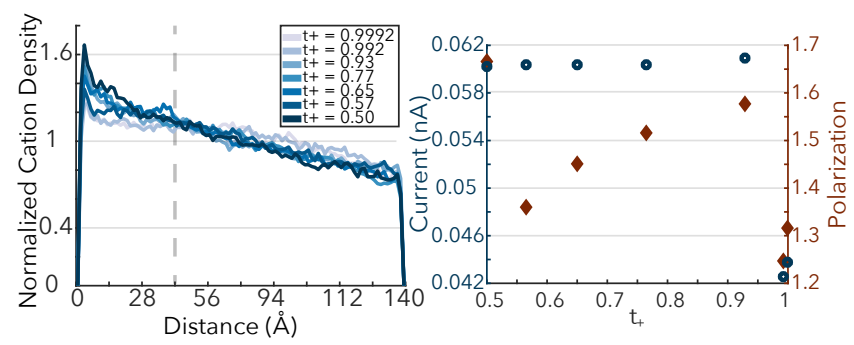

Figure 7: Left: Cation concentration profiles as a function of distance from the left foil anode over a range of transference numbers at fixed cation mobility. The grey dashed line indicates the boundary between the electrolyte and composite cathode regions. Right: Power density (blue circles) and polarization (orange diamonds) as a function of transference number for the same systems plotted to the left. Polarization is plotted as the maximum normalized cation density observed near the foil anode.

In contrast, figure 7 looks at a fixed cation mobility over a range of transference numbers. Figure 7 plots the concentration profiles at a constant $\mu_{+}=21.5 \mu \mathrm{m} \mathrm{V}^{-1} \mathrm{~s}^{-1}$, (i.e. the darkest orange line in Fig. 5) as well as the power density and maximum cation density in the same style as in Fig. 6. Despite the transference number spanning from 0.5 to 0.999 , the concentration profiles remain relatively constant in comparison to the scale of changes seen by varying the cation mobility. For very high transference numbers $(>0.99)$, the cation density could be interpreted as less polarized, consistent with predictions from macroscopic models. ${ }^{1}$ However, the steady-state power density is much lower than for moderate numbers (right panel), supporting the idea that the otherwise mobile cations are being slowed by their attractive interactions with the very slow anions, and breaking the expected trend between transference number, polarization, and battery performance. 
These simulations suggest that for ultrathin electrolyte systems, there is no clear benefit to optimizing the transference number in order to improve concentration polarization. Instead, concentration polarization is a function of the relative rates of reaction at the interface, and cation mobility. Additionally, there is some evidence that at very high transference numbers, battery performance is decreased due to the attractive interactions between ions with very little benefit to cation polarization, consistent with experimental results in macroscopic single ion conductor systems as well. ${ }^{17}$

\section{Acknowledgement}

This work was supported by the Toyota Research Institute. K. A. D. acknowledges support from the National Science Foundation (Graduate Research Fellowship Program). A. P. W. Acknowledges support from the Research Corporation for Scientific Advancement (Cottrell Scholar).

\section{References}

(1) Doyle, M.; Fuller, T. F.; Newman, J. Electrochimica Acta 1994, 39, 2073-2081.

(2) Diederichsen, K. M.; McShane, E. J.; McCloskey, B. D. ACS Energy Letters 2017, 2, 2563-2575.

(3) Newman, J.; Thomas-Alyea, K. E. Electrochemical Systems, 3rd ed.; Wiley - Interscience: Hoboken, NJ, 2004.

(4) Lu, Y.; Tikekar, M.; Mohanty, R.; Hendrickson, K.; Ma, L.; Archer, L. A. Advanced Energy Materials 2015, 5, 1-7.

(5) Cai, Z.; Liu, Y.; Liu, S.; Li, L.; Zhang, Y. Energy and Environmental Science 2012, 5, $5690-5693$. 
(6) Tikekar, M. D.; Archer, L. A.; Koch, D. L. Journal of the Electrochemical Society 2014, 161.

(7) Bruce, P. G.; Evans, J.; Vincent, C. A. Solid State Ionics 1988, 28-30, 918-922.

(8) Walls, H. J.; Zawodzinski, T. A. Electrochemical and Solid-State Letters 2000, 3, 321324.

(9) Evans, J.; Vincent, C. A.; Bruce, P. G. Polymer 1987, 28, 2324-2328.

(10) Balsara, N. P.; Newman, J. Journal of the Electrochemical Society 2015, 162, A2720A2722.

(11) Ma, Y.; Doyle, M.; Fuller, T. F.; Doeff, M. M.; De Jonghe, L. C.; Newman, J. Journal of the Electrochemical Society 1995, 142, 1859-1868.

(12) Chen, N.; Reeja-Jayan, B.; Lau, J.; Moni, P.; Liu, A.; Dunn, B.; Gleason, K. K. Materials Horizons 2015, 2, 309-314.

(13) Cao, Z.; Peng, Y.; Voth, G. a. The Journal of Physical Chemistry B 2015, 119, 75167521.

(14) Dwelle, K. A.; Willard, A. P. The Journal of Physical Chemistry C 2019, 123, 2409524103.

(15) Wright, M. R. An Introduction to Aqueous Electrolyte Solutions; John Wiley, 2007.

(16) Doyle, M. Journal of The Electrochemical Society 1993, 140, 1526.

(17) Zhang, H.; Li, C.; Piszcz, M.; Coya, E.; Rojo, T.; Rodriguez-Martinez, L. M.; Armand, M.; Zhou, Z. Chemical Society Reviews 2017, 46, 797-815. 